\title{
Mean of Other Group Imputation Technique
}

National Cancer Institute

\section{Source}

National Cancer Institute. Mean of Other Group Imputation Technique. NCI Thesaurus. Code C81204.

The substitution of missing data with the mean value from a comparator or reference group. 